Physics

Physics Research Publications

Purdue University

Year 2004

\title{
Three-body losses in trapped Bose-Einstein-condensed gases
}

\author{
Y. E. Kim \\ A. L. Zubarev
}

This paper is posted at Purdue e-Pubs.

http://docs.lib.purdue.edu/physics_articles/476 


\title{
Three-body losses in trapped Bose-Einstein-condensed gases
}

\author{
Yeong E. Kim* and Alexander L. Zubarev ${ }^{\dagger}$ \\ Purdue Nuclear and Many-Body Theory Group (PNMBTG), Department of Physics, Purdue University, \\ West Lafayette, Indiana 47907, USA \\ (Received 6 May 2003; published 3 February 2004)
}

\begin{abstract}
A time-dependent Kohn-Sham-like equation for $N$ bosons in a trap is generalized for the case of inelastic collisions. We derive adiabatic equations which are used to calculate the nonlinear dynamics of the BoseEinstein condensate and non-mean-field corrections due to the three-body recombination. We find that the calculated corrections are about 13 times larger for three-dimensional (3D) trapped dilute bose gases and about seven times larger for 1D trapped weakly interacting Bose gases when compared with the corresponding corrections for the ground-state energy and for the collective frequencies. The results are obtained at zero temperature.
\end{abstract}

DOI: 10.1103/PhysRevA.69.023602

PACS number(s): 03.75.Kk, 05.30.Jp

The newly created Bose-Einstein condensates (BECs) of weakly interacting alkali-metal atoms [1] stimulated a large number of theoretical investigations [2]. Most of these works are based on the assumption that the properties of BECs are well described by the Gross-Pitaevskii (GP) mean-field theory [3].

According to the theory of Lee, Huang, and Yang [4] the non-mean-field (NMF) correction to the GP theory behaves like $\sqrt{a^{3} n}$, where $n$ is the atomic density and $a$ is the $s$-wave scattering length of the interatomic interaction. The gas parameter $a^{3} n$ can have a large value when the number of atoms $N$ in the BEC or $a$ is large. In recent experiments [5], $N$ was of the order $10^{8}$ with an intermediate value of the gas parameter, $a^{3} n \approx 10^{-3}$.

Recently, it has become possible to tune the atomic scattering length to essentially any values, by exploiting the Feshbach resonances (FRs) [6-8]. We note that the FRs do not simply increase the gas parameter [9-14], and we do not consider FRs in this paper. Theoretical investigations of the NMF corrections to the ground state properties and to the collective frequencies of trapped BECs have already reported in the literature [15-19].

Inelastic collisions are an important issue in the physics of ultracold gases [20-23]. The main goal of this paper is to consider a nonlinear dynamics of the BEC due to three-body recombination. We calculate the corrections to the rate of the three-body recombination due to the NMF effects. These corrections are calculated in the large- $N$ limit and at zero temperature.

Our starting point is the Kohn-Sham (KS)-like equation [24] for $N$ interacting bosons in a trap potential $V_{\text {ext }}$,

$$
i \hbar \frac{\partial \Psi}{\partial t}=-\frac{\hbar^{2}}{2 m} \nabla^{2} \Psi+V_{\mathrm{ext}} \Psi+\frac{\partial[n \epsilon(n)]}{\partial n} \Psi,
$$

where $n(\vec{r}, t)=|\Psi(\vec{r}, t)|^{2}$, and $\epsilon(n)$ is the ground state energy per particle of a uniform system.

\footnotetext{
*Email address: yekim@ physics.purdue.edu

†Email address: zubareva@physics.purdue.edu
}

In Ref. [24], a rigorous proof is given to show that the KS-like equation correctly describes properties of the TonksGirardeau gas in general time-dependent harmonic trap in the large $N$ limit. The condition for the applicability of Eq. (1) demands that the function $\Psi$ changes slowly on distances of the order of the mean atomic separation $n^{-1 / 3}$. Since the characteristic distance of changing $\Psi$ for the trapped gas is of the order of the radius of the condensate $R$, one gets the condition $N \approx n R^{3} \gg 1$.

In order to take into account atoms lost by inelastic collisions (background collisions, dipolar relaxation, three-body recombination, etc.) we model the loss by the rate equation

$$
\frac{d N}{d t}=-\int \chi(\vec{r}, t) d \vec{r}
$$

where $\chi(\vec{r}, t)=\Sigma_{l=1} k_{l} n^{l} g_{l}(n), n^{l} g_{l}$ is the local $l$-particle correlation function, and $k_{l}$ is the rate constant for the $l$-body atoms loss. For atoms in BECs, this rate constant is reduced by a factor of $l$, which arises from the coherence properties of condensate $[25,26]$.

The generalization of Eq. (1) for the case of inelastic collisions reads

$$
\begin{aligned}
i \hbar \frac{\partial \Psi}{\partial t}= & -\frac{\hbar^{2}}{2 m} \nabla^{2} \Psi+V_{\text {ext }} \Psi+\frac{\partial[n \epsilon(n)]}{\partial n} \Psi \\
& -i \frac{\hbar}{2} \sum_{l=1} k_{l} n^{l-1} g_{l}(n) \Psi .
\end{aligned}
$$

Equation (3) is the main result of this paper. We will call Eq. (3) a dissipative KS-like equation. We note that Eq. (3) can be obtained from Eq. (1) by replacing $\epsilon(n)$ with $\epsilon(n)$ $-i \Gamma / 2$, where

$$
\Gamma / 2=\sum_{l=1}[\hbar /(2 n)] k_{l} \int_{0}^{n} x^{l-1} g_{l}(x) d x .
$$

It can be proved that every solution of the KS-like equation (1) is a stationary point of an action corresponding to the Lagrangian density 


$$
\mathcal{L}_{0}=\frac{i \hbar}{2}\left(\Psi \frac{\partial \Psi^{*}}{\partial t}-\Psi * \frac{\partial \Psi}{\partial t}\right)+\frac{\hbar^{2}}{2 m}|\nabla \Psi|^{2}+\epsilon(n) n+V_{\mathrm{ext}} n
$$

where the asterisk denotes the complex conjugation. Indeed, the substitution of $\mathcal{L}_{0}$ [Eq. (5)] into the Euler-Lagrange (EL) equations

$$
\begin{gathered}
\frac{\delta \mathcal{L}_{0}}{\delta \Psi^{*}}=\frac{\partial \mathcal{L}_{0}}{\partial \Psi^{*}}-\frac{\partial}{\partial t} \frac{\partial \mathcal{L}_{0}}{\partial\left(\partial \Psi^{*} / \partial t\right)}-\nabla \frac{\partial \mathcal{L}_{0}}{\partial\left(\nabla \Psi^{*}\right)}=0, \\
\frac{\delta \mathcal{L}_{0}}{\delta \Psi}=\frac{\partial \mathcal{L}_{0}}{\partial \Psi}-\frac{\partial}{\partial t} \frac{\partial \mathcal{L}_{0}}{\partial(\partial \Psi / \partial t)}-\nabla \frac{\partial \mathcal{L}_{0}}{\partial(\nabla \Psi)}=0
\end{gathered}
$$

gives Eq. (1) and its complex conjugate equation.

Now for the dissipative KS-like equation (3) we write the corresponding Lagrangian $\mathcal{L}$ as a sum of two terms, a conservative one $\mathcal{L}_{0}$, Eq. (4), and nonconservative one $\mathcal{L}^{\prime}, \mathcal{L}$ $=\mathcal{L}_{0}+\mathcal{L}^{\prime}[27-29]$. Now the EL equations read

$$
\begin{gathered}
\frac{\partial \mathcal{L}_{0}}{\partial \Psi^{*}}-\frac{\partial}{\partial t} \frac{\partial \mathcal{L}_{0}}{\partial\left(\partial \Psi^{*} / \partial t\right)}-\nabla \frac{\partial \mathcal{L}_{0}}{\partial\left(\nabla \Psi^{*}\right)}+\frac{\delta \mathcal{L}^{\prime}}{\delta \Psi^{*}}=0, \\
\frac{\partial \mathcal{L}_{0}}{\partial \Psi}-\frac{\partial}{\partial t} \frac{\partial \mathcal{L}_{0}}{\partial(\partial \Psi / \partial t)}-\nabla \frac{\partial \mathcal{L}_{0}}{\partial(\nabla \Psi)}+\frac{\delta \mathcal{L}^{\prime}}{\delta \Psi}=0
\end{gathered}
$$

A comparison Eqs. (6) with Eq. (3) gives

$$
\begin{aligned}
\frac{\delta \mathcal{L}^{\prime}}{\delta \Psi^{*}} & =\frac{\partial \mathcal{L}^{\prime}}{\partial \Psi^{*}}-\frac{\partial}{\partial t} \frac{\partial \mathcal{L}^{\prime}}{\partial\left(\partial \Psi^{*} / \partial t\right)}-\nabla \frac{\partial \mathcal{L}^{\prime}}{\partial\left(\nabla \Psi^{*}\right)} \\
& =-\frac{i \hbar}{2} \sum_{l=1} k_{l} n^{l-1} g_{l}(n) \Psi, \\
\frac{\delta \mathcal{L}^{\prime}}{\delta \Psi} & =\frac{\partial \mathcal{L}^{\prime}}{\delta \Psi}-\frac{\partial}{\partial t} \frac{\partial \mathcal{L}^{\prime}}{\partial(\partial \Psi / \partial t)}-\nabla \frac{\partial \mathcal{L}^{\prime}}{\partial(\nabla \Psi)} \\
& =\frac{i \hbar}{2} \sum_{l=1} k_{l} n^{l-1} g_{l}(n) \Psi^{*} .
\end{aligned}
$$

We are now ready to rewrite the Hamilton principle $\delta \int d t \int d \vec{r}\left(\mathcal{L}_{0}+\mathcal{L}^{\prime}\right)=0$ as

$$
\begin{aligned}
& \delta \int d t L_{0}+\frac{i \hbar}{2} \sum_{l=1} k_{l} \int d t \int d \vec{r} n^{l-1} g_{l}(n)\left(\Psi^{*} \delta \Psi-\Psi \delta \Psi^{*}\right) \\
& \quad=0
\end{aligned}
$$

where $L_{0}=\int \mathcal{L}_{0} d \vec{r}$. The variational formulation [Eq. (8)] is an extension of the standard variational formulation for the situation where a Lagrangian corresponding to the original equations cannot be found or does not exist [27-29].

For the remainder of this paper, we will focus and specialize solely on the three-body recombination. A suitable trial function can be taken as $\Psi(\vec{r}, t)$ $=\exp [-(i / \hbar) \phi(t)] \Phi(\vec{r}, t)$, where both $\phi$ and $\Phi$ are real functions. With this ansatz, the Hamiltonian principle [Eq. (8)] gives the following variational equations:

$$
\frac{d N}{d t}=-k_{3} \int \Phi^{6}(\vec{r}, t) g_{3}\left[\Phi^{2}(\vec{r}, t)\right] d \vec{r}
$$

and

$$
-\frac{\hbar^{2}}{2 m} \nabla^{2} \Phi+V_{\mathrm{ext}} \Phi+\frac{\partial[n \epsilon(n)]}{\partial n} \Phi=\mu(t) \Phi,
$$

where $N(t)=\int \Phi^{2}(\vec{r}, t) d \vec{r}$, and $\mu(t)=d \phi / d t$. The condition of the validity of the adiabatic equations (9) and (10) is $d N / d t<\omega_{\nu} N$, where $\omega_{\nu}$ is a frequency of elementary excitation.

The ground state energy per particle, $\epsilon(n)$, in the lowdensity regime can be calculated using an expansion in powers of $\sqrt{n a^{3}}$ :

$$
\begin{aligned}
\epsilon(n)= & \frac{2 \pi \hbar^{2}}{m} a n\left[1+\frac{128}{15 \sqrt{\pi}}\left(n a^{3}\right)^{1 / 2}\right. \\
& \left.+8\left(\frac{4 \pi}{3}-\sqrt{3}\right) n a^{3}\left[\ln \left(n a^{3}\right)+C\right]+\cdots\right] .
\end{aligned}
$$

The first term corresponding to the mean field prediction was first calculated by Lenz [30]. The second term was first calculated by Lee, Huang, and Yang [4], while the coefficient of the logarithm term was first obtained by $\mathrm{Wu}$ [31]. The constant $C$ after the logarithm term was considered in Ref. [32].

Expansion (11) is asymptotic, and it was shown in Ref. [33] that the Lee-Huang-Yang (LHY) correction [second term in Eq. (11)] represents a significant improvement on the mean field prediction up to $a^{3} n \approx 10^{-2}$, but the logarithmic correction already is wrong at $n a^{3} \approx 10^{-3}$. In Refs. [17-19], the LHY expansion [first two terms in expansion (11)] has been used to study effects beyond the mean field approximation.

We do not consider the logarithmic term in expansion (11), and rewrite Eq. (10) in the limit of large $N$ as

$$
\frac{m}{4 \pi a \hbar^{2}}\left(\mu-V_{\mathrm{ext}}\right)=n\left(1+\frac{32}{3 \sqrt{\pi}} \sqrt{n a^{3}}\right),
$$

where $n=\Phi^{2}(\vec{r}, t)$.

At densities $\left(n a^{3} \leqslant 10^{-3}\right)$ Eq. (12) can be solved by iteration,

$$
\begin{aligned}
n= & \frac{m}{4 \pi a \hbar^{2}}\left(\mu-V_{\mathrm{ext}}\right)-\frac{4 m^{3 / 2}}{3 \pi^{2} \hbar^{3}}\left(\mu-V_{\mathrm{ext}}\right)^{3 / 2}+\frac{32 a m^{2}}{3 \pi^{3} \hbar^{4}} \\
& \times\left(\mu-V_{\mathrm{ext}}\right)^{2}-\frac{896 a^{2} m^{5 / 2}}{9 \pi^{4} \hbar^{5}}\left(\mu-V_{\mathrm{ext}}\right)^{5 / 2}+\cdots
\end{aligned}
$$

where

$$
\begin{aligned}
\mu= & \mu_{\mathrm{TF}}\left[1+\frac{1}{2} \frac{a m^{1 / 2} \mu_{\mathrm{TF}}^{1 / 2}}{\hbar}-\left(\frac{1024}{105 \pi^{2}}-\frac{9}{16}\right) \frac{a^{2} m \mu_{\mathrm{TF}}}{\hbar^{2}}\right. \\
& \left.+\left(\frac{25}{32}-\frac{22}{21 \pi^{2}}\right) \frac{a^{3} m^{3 / 2} \mu_{\mathrm{TF}}^{3 / 2}}{\hbar^{3}}-\cdots\right],
\end{aligned}
$$


and the Thomas-Fermi (TF) approximation is simply obtained by keeping only the first term in the right side of Eq. (13), $n_{\mathrm{TF}}=m\left(\mu_{\mathrm{TF}}-V_{\mathrm{ext}}\right) /\left(4 \pi a \hbar^{2}\right)$.

Equation (13) holds in the region where $n$ is positive and $n=0$ outside this region. We note that the second term in Eq. (14) was considered in Refs. [15,19].

For the harmonic trap potential $V_{\text {ext }}(\vec{r})=(m / 2)\left(\omega_{x}^{2} x^{2}\right.$ $\left.+\omega_{y}^{2} y^{2}+\omega_{z}^{2} z^{2}\right), \quad \mu_{\mathrm{TF}}$ is given by [34] $\mu_{\mathrm{TF}}$ $=\hbar^{2} /\left(2 m a_{h o}^{12 / 5}\right)(15 a N)^{2 / 5}$, where $a_{h o}=\left(\hbar / m \omega_{h o}\right)^{1 / 2}$ is the oscillator length and $\omega_{h o}=\left(\omega_{x} \omega_{y} \omega_{z}\right)^{1 / 3}$. Equation (13) in this case becomes

$$
\begin{aligned}
\mu= & \mu_{\mathrm{TF}}\left[1+\frac{1}{2}\left[4 \pi a^{3} n_{\mathrm{TF}}(0)\right]^{1 / 2}-\left(\frac{1024}{105 \pi^{2}}-\frac{9}{16}\right)\right. \\
& \left.\times 4 \pi a^{3} n_{\mathrm{TF}}(0)+\left(\frac{25}{32}-\frac{22}{21 \pi^{2}}\right)\left[4 \pi a^{3} n_{\mathrm{TF}}(0)\right]^{3 / 2}-\cdots\right],
\end{aligned}
$$

where $n_{\mathrm{TF}}(0)=m \mu_{\mathrm{TF}} / 4 \pi a \hbar^{2}$.

Using the correlation function [26]

$$
g_{3}(n)=\left(1+\frac{64}{\sqrt{\pi}} \sqrt{n a^{3}}+\cdots\right),
$$

it can easily be seen that

$$
\begin{aligned}
\int \Phi^{6}(\vec{r}, t) g_{3}(n) d \vec{r}= & \frac{8}{21} n_{\mathrm{TF}}^{2}(0) N\left(1+\frac{3357}{512}\right. \\
& \left.\times\left[4 \pi a^{3} n_{\mathrm{TF}}(0)\right]^{1 / 2}+\cdots\right) .
\end{aligned}
$$

The solutions of Eqs. (9) and (10) in the mean-field approximation, corresponding to the first term in expansions (16) and (17), read

$$
\Phi^{2}(\vec{r}, t)=\frac{8}{21}\left(\frac{a^{6 / 5} a_{h o}^{24 / 5}}{\alpha N^{4 / 5}(0)}+\frac{4}{5} k_{3} t\right)^{-1 / 2}-\frac{m}{4 \pi a \hbar^{2}} V_{\mathrm{ext}}
$$

and

$$
N(t)=\left(N^{-4 / 5}(0)+\frac{4}{5} \frac{\alpha k_{3}}{a^{6 / 5} a_{h o}^{24 / 5}} t\right)^{-5 / 4},
$$

where $\alpha=15^{4 / 5} / 168 \pi^{2}$, and for the rate $\tau=|d \ln N / d t|$ we obtain

$$
\tau=\frac{\alpha k_{3} / a^{6 / 5} a_{h o}^{24 / 5}}{N^{-4 / 5}(0)+(4 / 5)\left(\alpha k_{3} / a^{6 / 5} a_{h o}^{24 / 5}\right) t} .
$$

Analytical results [Eqs. (18)-(20)] predict a strong $\omega_{\text {ho }}$ dependence of the three-body recombination in the TF regime, as shown for a ${ }^{87} \mathrm{Rb}$ condensate with $k_{3}$ $=5.8 \times 10^{-30} \mathrm{~cm}^{6} / \mathrm{s}[25]$ in Fig. 1. For all cases of Fig. 1, the adiabaticity is insured $\left(\dot{N} / N \leqslant 4 \times 10^{-4} \omega_{h o}\right.$ and $\dot{N} / N$ $\leqslant 5 \times 10^{-3} \omega_{h o}$ for $\omega_{h o} / 2 \pi=12.83$ and $77.78 \mathrm{~Hz}$, respectively). We expect that Eq. (3) has a validity when $\epsilon(n)$

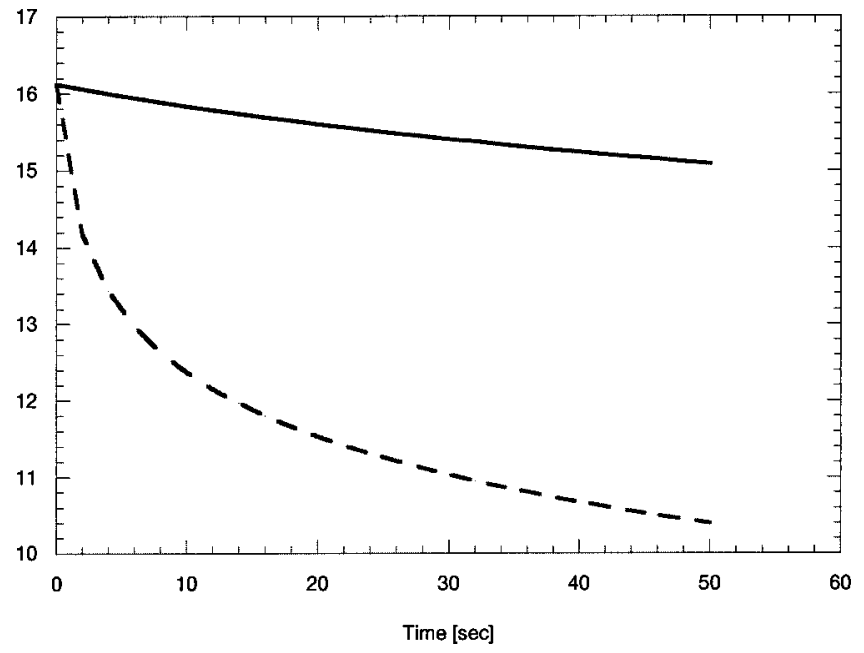

FIG. 1. The natural log of the number of ${ }^{87} \mathrm{Rb}$ atoms of the BEC in the trap as a function of time. The loss is due to the three-body recombination. Solid and dashed lines represent the theoretical results for traps with geometric averages of the oscillator frequencies $\omega_{h o} / 2 \pi=12.83 \mathrm{~Hz}$ and $\omega_{h o} / 2 \pi=77.78 \mathrm{~Hz}$, respectively.

$>\Gamma / 2$ [see Eq. (4)]. For the case of ${ }^{87} \mathrm{Rb}$ condensate with the gas parameter $a^{3} n \approx 10^{-3}, \Gamma /[2 \epsilon(n)] \approx 5 \times 10^{-4}$.

A comparison of Eq. (17) with Eq. (15) shows that the non-mean-field corrections to the nonlinear dynamics of the $\mathrm{BEC}$ due to the three-body recombination are about 13 times larger than corresponding corrections to the ground state and to the collective frequencies of trapped BECs. As an example, in Fig. 2 we present the results of numerical calculation of the non-mean-field corrections to the rate $\tau$ $=|d \ln N / d t|$ for the BECs of ${ }^{87} \mathrm{Rb}$ atoms with $N(0)=10^{8}$.

The LHY expansion is a major result of many-body theory, and direct observation of the NMF corrections "would represents an important achievement in the study of many-body effects associated with the Bose condensation"

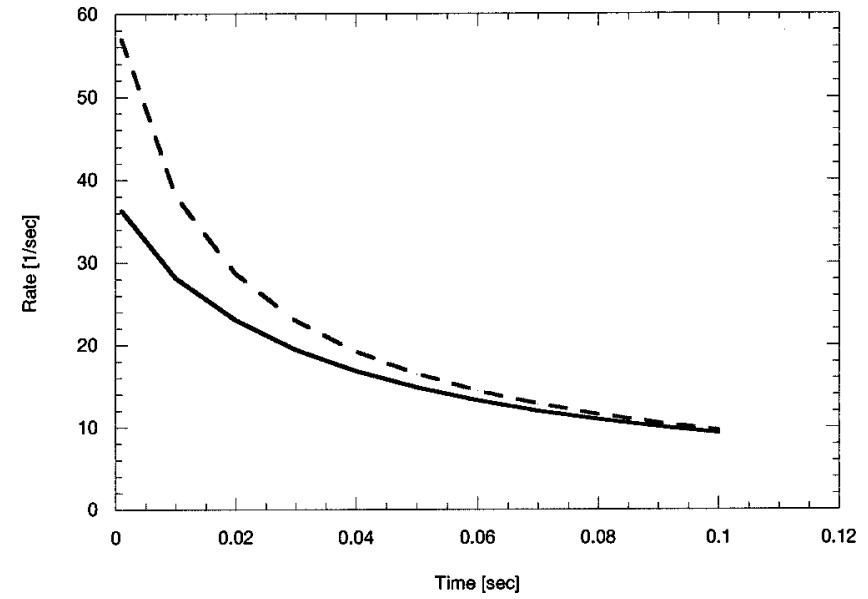

FIG. 2. Rate of the three-body recombination, $\tau=|d \ln N / d t|$, as a function of time for the BEC of ${ }^{87} \mathrm{Rb}$ atoms for the initial condition $N(t=0)=10^{8}$. The asymmetry parameter of the trap is $\lambda$ $=\sqrt{8}$, and $\omega_{z} /(2 \pi)=220 \mathrm{~Hz}$. The solid line corresponds to the mean-field approximation and the dashed line shows the results of inclusion of the non-mean-field corrections. 
[19]. The comparison of experiment [25] with our results shows that experimental uncertainties [25] are larger than calculated NMF corrections. To extract the NMF corrections, we need additional experimental data with smaller uncertainties.

The LHY expansion is no longer valid for cold Bose gases near Feshbach resonances. In this case, $\epsilon(n)$ can be found, for example, by method described in Ref. [9]. The effects of Feshbach resonances on nonlinear dynamics of the trapped BECs will be addressed in a future paper.

For a one-dimensional (1D) Bose gas interacting via a repulsive $\delta$-function potential, $\widetilde{g} \delta(x)$, the Lieb-Liniger (LL) model [35], $\epsilon(n)$ is given by [35] $\epsilon(n)=\left(\hbar^{2} / 2 m\right) n^{2} e(\gamma)$, where $\gamma=m \tilde{g} /\left(\hbar^{2} n\right)$ and for small values of $\gamma$ an expression for $\epsilon(n)$,

$$
\epsilon(n)=\frac{\tilde{g}}{2}\left(n-\frac{4}{3 \pi} \sqrt{\frac{m \widetilde{g} n}{\hbar^{2}}}+\cdots\right),
$$

is adequate up to approximately $\gamma=2$ [35]. In this case the large- $N$ limit solution of Eq. (10) reads

$$
n=\frac{\mu-V_{t}}{\widetilde{g}}+\frac{1}{\pi}\left(\frac{m \widetilde{g}}{\hbar^{2}}\right)^{1 / 2}\left(\frac{\mu-V_{t}}{\widetilde{g}}\right)^{1 / 2}+\cdots,
$$

where

$$
\mu=\mu_{\mathrm{TF}}\left(1-\frac{1}{4} \sqrt{\gamma(0)}+\cdots\right),
$$

with $\quad \gamma(0)=m \widetilde{g} / \hbar^{2} n(0)$ and for $V_{t}=m \omega^{2} x^{2} / 2, \quad \mu_{\mathrm{TF}}$ $=\left(3 N \widetilde{g} m^{1 / 2} \omega / 2^{5 / 2}\right)^{2 / 3}$. Using the 1D correlation function [36] $g_{3}^{1 \mathrm{D}}(n)=1-6 \sqrt{\gamma} / \pi+\cdots$, we obtain

$$
\int \Phi^{6}(x, t) g_{3}^{1 \mathrm{D}}(n) d x=\frac{24}{35} n_{\mathrm{TF}}^{2}(0) N\left(1-\frac{973}{512} \sqrt{\gamma(0)}+\cdots\right),
$$

where $n_{\mathrm{TF}}(0)=\mu_{\mathrm{TF}} / \widetilde{g}$.

Equation (23) shows that for the weak interacting trapped 1D bosons the NMF corrections to the three-body recombination dynamics are about seven times larger than corresponding corrections to the ground-state energy.

At temperature $T \ll \gamma \hbar^{2} n^{2} / 2 m$, thermal fluctuations provide only small corrections to the zero temperature $g_{3}^{1 \mathrm{D}}$ [36]. For temperatures $T \gg \gamma \hbar^{2} n^{2} / 2 m$ (but still $T \ll \sqrt{\gamma} \hbar^{2} n^{2} / 2 m$ ), $g_{3}^{1 \mathrm{D}}$ increases with increasing temperature [37].

Finally, we mention the modified many-body $T$-matrix theory $[38,39]$. This theory gives an explanation of the experimentally shown reduction of the three-body recombination in the 2D homogeneous atomic hydrogen gas at finite temperatures [40]. In our future work, we will consider 2D trapped Bose gases.

In conclusion, we have developed a time-dependent dissipative KS-like equation for $N$ bosons in a trap for the case of inelastic collisions. We derive adiabatic equations which are used to calculate the nonlinear dynamics of the BEC due to the three-body recombination. The calculated non-meanfield corrections to the three-body recombination dynamics are shown to be about 13 times larger for 3D trapped dilute Bose gases and about seven times larger for 1D trapped weakly interacting Bose gases than corresponding corrections to the ground state energy and to the collective frequencies. The results are obtained at zero temperature.

We thank S. Khlebnikov for his interest and comments.
[1] Bose-Einstein Condensation in Atomic Gases, Proceedings of the International School of Physics "Enrico Fermi," edited by M. Inguscio, S. Stringari, and C. E. Wieman (IOS Press, Amsterdam, 1999); http://amo.phy.gasou.edu/bec.html; http:// jilawww.colorado.edu/bec/ and references therein.

[2] A. L. Fetter and A. A. Svidzinsky, J. Phys.: Condens. Matter 13, R135 (2001); A. J. Leggett, Rev. Mod. Phys. 73, 307 (2001); K. Burnett, M. Edwards, and C. W. Clark, Phys. Today 52(12), 37 (1999); F. Dalfovo, S. Giorgini, L. Pitaevskii, and S. Stringari, Rev. Mod. Phys. 71, 463 (1999); S. Parkins and D. F. Walls, Phys. Rep. 303, 1 (1998).

[3] L. P. Pitaevskii, Zh. Eksp. Teor. Fiz. 40, 646 (1961) [Sov. Phys. JETP 13, 451 (1961)]; E. P. Gross, Nuovo Cimento 20, 454 (1961); J. Math. Phys. 4, 195 (1963).

[4] T. D. Lee and C. N. Yang, Phys. Rev. 105, 1119 (1957); T. D. Lee, K. W. Huang, and C. N. Yang, ibid. 106, 1135 (1957).

[5] W. Ketterle, D. S. Durfee, and D. M. Stamper-Kurn, in BoseEinstein Condensation in Atomic Gases (Ref. [1]), p. 67; D. M. Stamper-Kurn, H.-J. Miesner, S. Inouye, M. R. Andrews, and W. Ketterle, Phys. Rev. Lett. 81, 500 (1998).

[6] S. Inouye, M. R. Andrews, J. Stenger, H. J. Miesner, D. M. Stamper-Kurn, and W. Ketterle, Nature (London) 392, 151 (1998); P. Courteille, R. S. Freeland, D. J. Heinzen, F. A. van
Abeelen, and B. J. Verhaar, Phys. Rev. Lett. 81, 69 (1998); J. L. Roberts, N. R. Claussen, J. P. Burke, C. H. Greene, E. A. Cornell, and C. E. Wieman, ibid. 81, 5109 (1998); D. M. Stamper-Kurn, and W. Ketterle, ibid. 82, 2422 (1999); J. L. Roberts, N. R. Claussen, S. L. Cornish, E. A. Donley, E. A. Cornell, and C. E. Wieman, ibid. 86, 4211 (2001); E. A. Donley, N. R. Claussen, S. L. Cornish, J. L. Roberts, E. A. Cornell, and C. E. Wieman, Nature (London) 412, 295 (2001).

[7] S. L. Cornish, N. R. Claussen, J. L. Roberts, E. A. Cornell, and C. E. Wieman, Phys. Rev. Lett. 85, 1795 (2000).

[8] J. Stenger, S. Inouye, M. R. Andrews, H.-J. Meisner, D. M. Stamper-Kurn, and W. Ketterle, Phys. Rev. Lett. 82, 2422 (1999).

[9] Y. E. Kim and A. L. Zubarev, Phys. Lett. A 312, 277 (2003).

[10] J. H. Macek, Few-Body Syst. 31, 241 (2002).

[11] E. Timmermans, P. Tommasini, M. Hussein, and A. Kerman, Phys. Rep. 315, 199 (1999).

[12] V. A. Yurovsky, A. Ben-Reuven, P. S. Julienne, and C. J. Williams, Phys. Rev. A 60, R765 (1999).

[13] F. A. van Abeelen and B. J. Verhaar, Phys. Rev. Lett. 83, 1550 (1999).

[14] P. D. Drummond, K. V. Kheruntsyan, and H. He, Phys. Rev. Lett. 81, 3055 (1998); K. V. Kheruntsyan and P. D. Drum- 
mond, Phys. Rev. A 58, 2488 (1998); 58, R2676 (1998); 61, 063816 (2000).

[15] E. Timmermans, P. Tommasini, and K. Huang, Phys. Rev. A 55, 3645 (1997).

[16] E. Braaten and A. Nieto, Phys. Rev. B 56, 14745 (1997); E. Braaten and J. Pearson, Phys. Rev. Lett. 82, 255 (1999).

[17] A. Fabrocini and A. Polls, Phys. Rev. A 60, 2319 (1999); 64, 063610 (2001).

[18] A. Banerjee and M. P. Singh, Phys. Rev. A 64, 063604 (2001); 66, 043609 (2002).

[19] L. Pitaevskii and S. Stringari, Phys. Rev. Lett. 81, 4541 (1998).

[20] P. O. Fedichev, M. W. Reynolds, and G. V. Shlyapnikov, Phys. Rev. Lett. 77, 2921 (1996).

[21] E. Nielsen and J. H. Macek, Phys. Rev. Lett. 83, 1566 (1999).

[22] B. D. Esry, C. H. Greene, and J. P. Burke, Phys. Rev. Lett. 83, 1751 (1999).

[23] P. F. Bedaque, E. Braaten, and H.-W. Hammer, Phys. Rev. Lett. 85, 908 (2000); E. Braaten and H.-W. Hammer, ibid. 87, 160407 (2001).

[24] Y. E. Kim and A. L. Zubarev, Phys. Rev. A 67, 015602 (2003).

[25] E. A. Burt, R. W. Ghrist, C. J. Myatt, M. J. Holland, E. A. Cornell, and C. E. Wieman, Phys. Rev. Lett. 79, 337 (1997).

[26] Y. Kagan, B. V. Svistunov, and G. V. Shlyapnikov, Pis'ma Zh. Eksp. Teor. Fiz. 42, 169 (1985) [JETP Lett. 42, 209 (1985)].
[27] D. Anderson, A. Bondeson, and M. Lisak, Phys. Lett. A 67, 331 (1978).

[28] A. Bondeson, M. Lisak, and D. Anderson, Phys. Scr. 20, 479 (1979).

[29] S. Chavez Cerda, S. B. Cavalcanti, and J. M. Hickmann, Eur. Phys. J. D 1, 313 (1998).

[30] W. Lenz, Z. Phys. 56, 778 (1929).

[31] T. T. Wu, Phys. Rev. 115, 1390 (1959).

[32] E. Braaten, H.-W. Hammer, and T. Mehen, Phys. Rev. Lett. 88, 040401 (2002), and references therein.

[33] S. Giorgini, J. Boronat, and J. Casulleras, Phys. Rev. A 60, 5129 (1999).

[34] G. Baym and C. Pethick, Phys. Rev. Lett. 76, 6 (1996).

[35] E. H. Lieb and W. Liniger, Phys. Rev. 130, 1605 (1963).

[36] D. M. Gangardt and G. V. Shlyapnikov, Phys. Rev. Lett. 90, 010401 (2003).

[37] D. M. Gangardt and G. V. Shlyapnikov, New J. Phys. 5, 79 (2003).

[38] J. O. Andersen, U. Al. Khawaja, and H. T. C. Stoof, Phys. Rev. Lett. 88, 070407 (2002).

[39] U. Al. Khawaja, J. O. Andersen, N. P. Proukakis, and H. T. C. Stoof, Phys. Rev. A 66, 013615 (2002).

[40] A. I. Safonov, S. A. Vasilyev, I. S. Yasnikov, I. I. Lukashevich, and S. Jaakkola, Phys. Rev. Lett. 81, 4545 (1998). 